\title{
The effect of delayed presentation and surgery in pelvic trauma on morbidity and mortality
}

\author{
Wayne Hoskins ${ }^{1,2 *}$, Rohan Sheehy ${ }^{1}$, Andrew Bucknill ${ }^{1}$, Roselyn Santos ${ }^{3}$, Rodney Judson ${ }^{3}$, Kellie Gumm ${ }^{3}$ and Xavier Griffin $^{1}$ \\ ${ }^{1}$ Department of Orthopaedic Surgery, Royal Melbourne Hospital, Parkville, Victoria, Australia \\ ${ }^{2}$ Faculty of Medicine, Dentistry and Health Sciences, The University of Melbourne, Parkville, Victoria Australia \\ ${ }^{3}$ Trauma Service, Royal Melbourne Hospital, Parkville, Victoria, Australia
}

\begin{abstract}
Introduction: The presentation of pelvic trauma patients can be time critical. This study will aim to identify the impact of delayed presentation in pelvic trauma patients on morbidity and mortality and identify the effect of time to pelvic surgery on patient outcomes.

Methods: Patients presenting to a Level 1 Trauma Centre between July 2001 and June 2014 with major pelvic trauma were retrospectively identified using two prospective databases. Time from injury to arrival and surgery and referral from another hospital were identified. Outcomes included intensive care (ICU) admission, length of stay and mortality.

Results: 1300 patients were identified. $133(10.2 \%)$ patients were transferred from a rural hospital. The risk of death was higher in patients presenting directly (11.6\% vs. $6.1 \%, \mathrm{p}=0.028)$, although their Injury Severity Score was higher (28.1 vs. $24.0, \mathrm{p}<0.001)$. There was no difference in ICU days (3.95 vs. 3.58 , $\mathrm{p}=0.50)$ or length of stay $(14.97$ vs. $15.81, p=0.50)$. Transfer was more timely if ICU was required ( 17.9 vs. 46.8 hours, $\mathrm{p}=0.028)$. Pelvic surgery occurred in 79 ( $43.9 \%)$ in the transferred group and $370(33.0 \%)$ patients presenting directly.

Conclusions: Mortality is not increased with initial presentation at rural hospitals, although these patients had less severe injury. There is a higher mortality with earlier surgery, although this likely reflects the seriousness of the patient's condition rather than the surgery itself.
\end{abstract}

\section{Introduction}

The presentation of pelvic trauma can be time critical given the association with high energy trauma, haemodynamic instability and life threatening injuries $[1,2]$. The mortality risk from pelvic fracture is $19 \%[1,3,4]$, but as high as $37 \%$ with haemodynamic instability [5]. However, there has been little research to establish and quantify the link between presentation of pelvic trauma with morbidity and mortality. Continued advances in prehospital care, management of haemorrhage, trauma management and operative intervention have been thought to result in a reduction of mortality and morbidity [6], yet this has not been clearly documented in scientific literature.

Patients with pelvic fractures should initially be managed by Advanced Trauma Life Support (ATLS) principles [7]. Application of pelvic binder [8] can help control arterial and venous bleeding and bleeding from the pelvic bones [9]. Hypotensive patients are at significant higher risk of mortality [3. Surgical options with ongoing, unresponsive haemodynamic instability include angioembolisation, external fixation, internal fixation, direct surgical haemostasis and pelvic packing $[10,11]$. Which option is centre dependent [6], with no clear evidence for an optimal protocol [3]. However, recent data has shown that mortality decreases with the implementation of a protocol emphasizing early rapid and appropriate use of pelvic angiography [12]. Angioembolisation is not available in rural hospitals [6].

Currently, the Victorian State Trauma Guidelines specify that for non-trauma hospitals any patient with a suspected fractured pelvis should be triaged to the highest level of trauma service within
30 minutes $[13,14]$. If transport is greater than 30 minutes or there is an immediate life-threatening situation, transfer should occur to the nearest designated trauma service. Rural hospitals in Victoria are typically Level 3 Trauma centres and act as regional trauma a service that provide resuscitation and stabilization of major trauma patients prior to their transfer to a major trauma service and provides definitive care when transfer is not required [15]. In Victoria all rural hospitals are located between 90 minutes and 4 hours by road from Royal Melbourne Hospital (RMH), one of two adult Level 1 Trauma Centre's in the state.

This study aims to identify the impact delayed presentation has on morbidity and mortality in pelvic trauma patients. It will examine the effect of initial presentation at a rural or other metropolitan non-Level 1 Trauma Centre prior to definitive management at a Level 1 Trauma Centre. Finally this study will identify the effect of time to pelvic surgery on patient outcomes.

\section{Methods}

A retrospective review of patients with major pelvic trauma

Correspondence to: Wayne Hoskins, Royal Melbourne Hospital, Grattan St, Parkville VIC 3052 Australia, Tel: +613-9342-8479; Fax: +613-9342-8780; E-mail: waynehoskins@icloud.com

Key words: pelvic trauma, morbidity, mortality

Received: October 02, 2016; Accepted: October 25, 2016; Published: October 28, 2016 
presenting to RMH between July 2001 to July 2014 was identified using prospective databases, the RMH Trauma Registry. Patients were identified using Abbreviated Injury Scale (AIS) codes for pelvic fractures. The database records all trauma admissions with length of stay $>24$ hours. Major pelvic trauma was defined as patients with pelvic fracture and if one of the following criteria is met: Injury Severity Score (ISS) $>12$, intensive care unit (ICU) admission $>24$ hours requiring mechanical ventilation, urgent surgery $<48$ hours or death after injury [16]. It was identified whether patients initially presented to a rural or other hospital prior to transfer to RMH. Patients transferred from interstate or international hospitals were excluded. Time taken from injury to presentation and time to definitive pelvic surgery was recorded. Definitive pelvic surgery was defined as the final pelvic operation during inpatient stay. Patient demographics, injury details and severity, ambulance and referring hospital data, inpatient data and surgery details were analyzed. Severe pelvic fractures were defined as AIS Injury code severity $\geq 4$. Outcome measures were inpatient mortality, ICU requirement and length of stay and length of hospital stay. Descriptive statistics were used and differences between groups compared with independent t-tests, Chi Squared and Fisher's exact testing. For each, $\mathrm{p}<0.05$ was considered significant. Minimum follow up was 12 months. Approval was received from the RMH Human Research and Ethics Committee: QA2014123.

\section{Results}

There were a total of 1300 patients with $180(13.8 \%)$ transferred from another hospital; 133 (10.2\%) from a rural hospital and 47 (3.6\%) from another metropolitan hospital. Patients most commonly presented by road ambulance $(74.9 \%)$, then helicopter (17.3\%) and fixed wing (1.2\%). Table 1 compares data between those patients transferred and those presenting directly. The mean ISS for patients who died was 39.9. There were 11 deaths in the transferred patient group (6.1\%) compared with 130 in patients presenting directly (11.6\%), (RR 0.527 95\% CI $0.291,0.955, \mathrm{p}=0.028$ ).

For patients presenting directly to RMH, mortality was associated with a delayed time to initial presentation to the hospital (3.4 vs. 2.2 hours, $\mathrm{p}=0.006$ ). However, a quicker presentation was not associated with a reduction in ICU requirements $(\mathrm{p}=0.57)$ or ICU length of stay $(\mathrm{p}=0.87)$. For patients transferred from another hospital, mortality was not associated with transfer time ( 30.5 vs. 33.8 hours $\mathrm{p}=0.897$ ). For transferred patients requiring ICU admission, there was a quicker transfer time ( 17.9 vs. 46.8 hours, $\mathrm{p}=0.028$ ). Overall length of stay did not correlate with timing of arrival at $\mathrm{RMH}(\mathrm{p}=0.57)$. For patients requiring pelvic surgery, there was an increase in mortality with shorter time to definitive surgery ( 1.36 days vs. 5.05 days, $\mathrm{p}=0.013$ ).

\section{Patients managed surgically}

There were a total of 449 patients managed surgically, 79 (43.9\%) in the transferred group and $370(33.0 \%)$ patients presenting directly. Table 2 compares data between these two groups. There was no difference in time to surgery between groups (5.39 vs. 4.84 days). There were no deaths in the group of patients transferred and $11(3.0 \%)$ in the patients transferred directly $(\mathrm{p}=0.225)$. The ICU admission risk was $38.0 \%$ in the transferred group and $48.9 \%$ in the patients presenting directly $(\mathrm{p}=0.134)$. Nine of the 11 deaths had external fixation as their definitive pelvic surgery.

\section{Patients with severe pelvic fractures}

There were 272 patients with severe pelvic fractures, 235 (86.4\%) presenting directly and $37(13.6 \%)$ in the transferred group. Table 3 compares data between these two groups. Excluding deaths prior, $8 / 37$ (21.6\%) of transferred patients and 100/223 (44.8\%) of patients presenting directly had pelvic surgery on the day of admission $(\mathrm{p}=0.010)$. There was a difference in ICU requirements, with $15 / 35$ $(42.8 \%)$ of transferred patients and 135/210 (64.3\%) of patients presenting directly requiring ICU admission $(\mathrm{p}=0.030)$. The mortality rate for transferred patients was $2 / 37$ (5.4\%) compared with 51/235 $(21.7 \%)$ for patients presenting directly $(\mathrm{p}=0.011)$.

\section{Angioembolisation}

One hundred and five (8.1\%) patients received angioembolisation, with $31(29.5 \%)$ patients dying following this procedure. Only three patients transferred from a rural hospital received angioembolisation and one from another metropolitan hospital. The mean ISS for patients that died and received angioembolisation was 47.8 .

\section{Discussion}

This study showed that one in ten major pelvic trauma patients will

Table 1. Comparison of data between those patients transferred and those directly presenting.

\begin{tabular}{|l|l|l|l|}
\hline & $\begin{array}{l}\text { Transferred patients } \mathrm{n}=180 \text { (mean, } \\
\text { standard deviation) }\end{array}$ & $\begin{array}{l}\text { Direct presentation } \mathrm{n}=1120 \text { (mean, standard } \\
\text { deviation) }\end{array}$ & Mean difference $(95 \% \mathrm{CI})$ \\
\hline Injury Severity Score & $23.96(11.35)$ & $28.01(13.23)$ & $-4.044(-6.091,-1.998)$ \\
\hline Days in ICU & $3.58(6.45)$ & $3.95(6.76)$ & $-0.365(-1.423,0.693)$ \\
\hline Length of stay (days) & $15.81(12.92)$ & $14.97(15.92)$ & $-0.83918(-1.609,3.287)$ \\
\hline
\end{tabular}

Table 2. Comparison of data between those patients transferred and those directly presenting managed surgically.

\begin{tabular}{|l|l|l|l|}
\hline & $\begin{array}{l}\text { Transferred patients n=79 (mean, standard } \\
\text { deviation) }\end{array}$ & $\begin{array}{l}\text { Direct presentation } \mathrm{n}=370 \text { (mean, standard } \\
\text { deviation) }\end{array}$ & P value (95\% CI) \\
\hline Injury Severity Score & $21.39(10.7)$ & $28.11(13.7)$ & $<0.001(-9.943--3.494)$ \\
\hline Days in ICU & $3.04(5.3)$ & $5.30(8.4)$ & $0.023(-4.209--.321)$ \\
\hline Length of stay (days) & $17.43(14.3)$ & $19.75(17.9)$ & $0.28(-6.528--1.895)$ \\
\hline
\end{tabular}

Table 3. Comparison of data between those patients transferred and those directly presenting with severe pelvic fractures (AIS Injury code severity $\geq 4$ ).

\begin{tabular}{|l|l|l|l|}
\hline & $\begin{array}{l}\text { Transferred patients } \mathrm{n}=37 \text { (mean, standard } \\
\text { deviation) }\end{array}$ & $\begin{array}{l}\text { Direct presentation } \mathrm{n}=235 \text { (mean, standard } \\
\text { deviation) }\end{array}$ & P value (95\% CI) \\
\hline Injury Severity Score & $30.5(13.4)$ & $37.6(14.3)$ & $0.005(-11.855--2.250)$ \\
\hline Days in ICU & $3.3(5.1)$ & $5.6(7.7)$ & $0.019(-4.283--0.399)$ \\
\hline Length of stay (days) & $15.7(13.8)$ & $20.4(21.6)$ & $0.082(-10.038--0.608)$ \\
\hline
\end{tabular}


present initially to a rural hospital. These patients have approximately half the risk of mortality but lower ISS. Mortality was not associated with transfer time. Transferred patients had the same length of stay, risk of ICU admission and time spent in ICU than patients who presented directly. Patients who required ICU were transferred faster. Angioembolisation rarely occurred for transferred patients. Half of patients transferred were managed surgically compared with one third of patients presenting directly. The ISS of transferred patients requiring surgery was less. There was no difference in time to surgery between groups. For patients presenting directly there is a reduction in mortality with more timely arrival, but time of arrival did not change ICU requirements. Overall the mortality risk following pelvic surgery was low, with most surgical procedures being external fixation and mostly likely damage control measures which ended up being definitive. Subanalysis of patients with severe pelvic fractures showed that transferred patients had lower ISS and fewer requirements for immediate surgery on presentation and lower mortality risk.

Specific literature on time of presentation and time to theatre on major pelvic trauma is lacking. With rural general trauma, time from injury to definitive care is known to be prolonged [17], with a $19 \%$ increased risk of death per hour of time for ambulance transfer [18]. Research from Western Australia has shown that if the patient survives transfer to a Level 1 Trauma Centre, then mortality outcomes are equivalent to metropolitan trauma [19], which is supported by this study. The number of patients presenting by either helicopter or fixed wing (18.5\%) was higher than the total number of patients transferred from rural hospitals $(10.3 \%)$. This figure does not include arrival via road. As such it is not clear how many patients directly bypassed a rural hospital despite Victorian State Trauma Guidelines recommending initial presentation there. All these factors make drawing definitive conclusions on the success of the guidelines and determining whether best management occurs thorough direct admission to a Level 1 Trauma Centre versus earlier and temporary management at a rural hospital vexed.

In reviewing the data and interpreting the applicability of the Victorian State Trauma Guidelines, Victoria is a smaller state than elsewhere in Australia and rural Victoria typically has combined general/trauma surgery and orthopaedic surgery. Most of the available Australian literature on mortality for trauma transfers from rural centers is largely from Western Australia $[18,19]$ a state with more remote areas that typically only have general/trauma surgery access without orthopaedics. Accordingly the Victorian State Trauma Guidelines may not be directly transferable and other states may need to make appropriate modifications to suit their geography and surgical services provided.

Whilst the mortality rate for patients initially presenting to RMH appeared higher than transferred patients, this is not surprising given that their ISS scores were higher and patients may have bypassed a rural hospital. The ISS scores reported are similar to those that have been published elsewhere. McMurty et al. [4] found that the average ISS pelvic trauma patient is 33.5. Overall the mortality rate for patients requiring pelvic surgery was lower than figures previously reported in the literature $[1,3,4]$, as was the case for haemodynamic patients receiving angioembolisation [5]. When solely looking at patients with severe pelvic fractures, our mortality rate of $19.5 \%$ was similar to that reported in the literature. Our angioembolisation rate was almost exactly the same as the $9 \%$ that has recently been reported in a large series at a Level 1 Trauma Centre [20]. The higher mortality rate found with earlier definitive pelvic surgery is likely related to the fact that these patients were unwell and the surgery was damage control in nature, which ended up being definitive. In support of this, for 9/11 deaths, the definitive pelvic surgery was external fixation. It appears that timing of surgery was appropriate, with surgery occurring at a time frame once patients would have been physiologically optomised [21].

There are limitations in the study. Notably, there is a high association with other life threatening injuries in pelvic trauma [4]. These may have concurrently impacted on patient outcomes and timing of surgery is often dictated by associated injuries. Along with associated injuries we did not factor in patient physiology, age or comorbidity. These are likely confounding variables. We also do not have data for patients who died at the scene, prior to transfer or on the management provided at rural hospitals. Without this, it is not fully possible to critique the outcomes at these hospitals and comment on the true success of the pre-hospital triaging. Timing of surgery may not be related to severity as it could be driven by surgeon's availability, the nature of the injury and the need for surgery for other reasons unrelated to pelvic injury. It is most probable that patients who were rushed to RMH bypassing other hospitals were likely to be sicker than those surviving in a Level 3 trauma hospital for secondary transfer. The higher severity of injured patients and the pelvic trauma at a Level 1 Trauma Centre produces a selection bias when comparing outcomes to rural hospitals. In recording time to surgery, we recorded time from presentation to the hospital. Time between injury and surgery may have been more valuable, but this was not recorded and unable to be analyzed. We also did not examine how specific pelvic fracture types impacted on outcome, although we attempted to analyze this by looking at patients with severe pelvic fractures defined as AIS Injury code severity $\geq 4$. Lastly this is data from a single institution and the generalizability of findings may be limited.

\section{Conclusion}

Presentation of major pelvic trauma occurs at rural hospitals. This does not increase their risk of mortality or time to surgery when transferred to a Level 1 Trauma Centre, although these patients had less severe injury and data is not available for patients who died at the scene, prior to transfer or on the management provided at rural hospitals. Delayed direct presentation to a Level 1 Trauma Centre is associated with a higher mortality. There is a higher mortality with earlier surgery, although this likely reflects the seriousness of the patient's condition rather than the surgery itself.

\section{References}

1. Adams JE, Davis GG, Alexander CB, Alonso JE (2003) Pelvic trauma in rapidly fatal motor vehicle accidents. J Orthop Trauma 17: 406-410. [Crossref]

2. Poole GV, Ward EF, Muakkassa FF, Hsu HS, Griswold JA, et al. (1991) Pelvic fracture from major blunt trauma. Outcome is determined by associated injuries. Ann Surg 213: 532-538. [Crossref]

3. Gabbe BJ, de Steiger R, Esser M, Bucknill A, Russ MK, et al. (2011) Predictors of mortality following severe pelvic ring fracture: results of a population-based study. Injury 42: 985-991. [Crossref]

4. McMurtry R, Walton D, Dickinson D, Kellam J, Tile M (1980) Pelvic disruption in the polytraumatized patient: a management protocol. Clin Orthop Relat Res 22-30. [Crossref]

5. Heetveld MJ, Harris I, Schlaphoff G, Balogh Z, D'Amours SK, et al. (2004) Hemodynamically unstable pelvic fractures: recent care and new guidelines. World $J$ Surg 28: 904-909. [Crossref]

6. Wong JM, Bucknill A (2013) Fractures of the pelvic ring. Injury. [Crossref]

7. Advanced trauma life support course: student manual. 8th ed. Chicago: American College of Surgeons; 2008. 
8. Flint LM Jr, Brown A, Richardson JD, Polk HC (1979) Definitive control of bleeding from severe pelvic fractures. Ann Surg 189: 709-716. [Crossref]

9. Guthrie HC, Owens RW, Bircher MD (2010) Fractures of the pelvis. J Bone Joint Surg $\operatorname{Br}$ 92: 1481-1488. [Crossref]

10. Giannoudis PV, Pape HC (2004) Damage control orthopaedics in unstable pelvic ring injuries. Injury 35: 671-677. [Crossref]

11. Halawi MJ (2015) Pelvic ring injuries: Emergency assessment and management. J Clin Orthop Trauma 6: 252-258. [Crossref]

12. Black S, Sathy A, Jo C, Wiley M, Minei J, et al. (2016) Improved survival after pelvic fracture: 13-year experience at a single trauma center using a multidisciplinary institutional protocol. J Orthop Trauma 30: 22-28.

13. Cameron PA, Gabbe BJ, Cooper DJ, Walker T, Judson R, et al. (2008) A statewide system of trauma care in Victoria: effect on patient survival. Med J Aust 189: 546-550. [Crossref]

14. Victorian State Trauma System. Available at www.health.vic.gov.au/trauma/majortrauma.htm. Department of Health and Human Services, State Government of Victoria, Australia. Last updated 22 May, 2014. Accessed 02 May 2015.
15. Regional and rural trauma services. State Government of Victoria. 2010

16. Department of Human Services. Review of Trauma and Emergency Services 1999: final Report. Department of Human Services, Victoria. 1999.

17. Rogers FB, Shackford SR, Osler TM, Vane DW, Davis JH (1999) Rural trauma: the challenge for the next decade. J Trauma 47: 802-821. [Crossref]

18. Fatovich DM, Jacobs IG (2009) The relationship between remoteness and trauma deaths in Western Australia. J Trauma 67: 910-914. [Crossref]

19. Fatovich DM, Phillips M, Jacobs IG (2011) A comparison of major trauma patients transported to trauma centres vs. non-trauma centres in metropolitan Perth. Resuscitation 82: 560-563.

20. Lustenberger T, Wutzler S, Störmann P, Laurer H, Marzi I (2015) The role of angioembolization in the acute treatment concept of severe pelvic ring injuries. Injury 46 Suppl 4: S33-38. [Crossref]

21. Pape HC, Giannoudis PV, Krettek C (2002) The timing of fracture treatment in polytrauma patients: relevance of damage control orthopaedic surgery. Am J Surg 183: 622-629.

Copyright: (2016 Hoskins H. This is an open-access article distributed under the terms of the Creative Commons Attribution License, which permits unrestricted use, distribution, and reproduction in any medium, provided the original author and source are credited. 